\title{
Near-infrared variability of a sample of galactic carbon Miras ${ }^{\star \star \star}$
}

\author{
F. Kerschbaum ${ }^{1}$, M. A. T. Groenewegen ${ }^{2}$, and C. Lazaro ${ }^{3,4}$ \\ 1 Institute for Astronomy, University of Vienna, Türkenschanzstrasse 17, 1180 Vienna, Austria \\ e-mail: franz.kerschbaum@univie.ac.at \\ 2 Instituut voor Sterrenkunde, KU Leuven, Celestijnenlaan 200B, 3001 Heverlee, Belgium \\ 3 Dpto. de Astrofísica, Fac. de Física, Universidad de La Laguna, 38200 La Laguna, Tenerife, Spain \\ 4 Instituto de Astrofísica de Canarias, 38200-La Laguna, Tenerife, Spain
}

Received 9 January 2006 / Accepted 16 July 2006

\begin{abstract}
Aims. In this paper we aim to determine the longest pulsation period of infrared carbon stars. Methods. Forty-seven infrared carbon stars were selected based on (1) IRAS colours and spectral classification from the IRAS LRS atlas, and (2) known carbon stars with large CO expansion velocities. Multi-epoch $J H K L^{\prime}$ photometry was obtained. Results. Reliable periods could be derived for 31 stars. The two longest periods are 840 and 870 days, only slightly longer than the previously longest known period for a galactic carbon star of 783 days. This is considerably shorter than the periods of some $\mathrm{OH} / \mathrm{IR}$ stars. As the present survey targeted carbon stars that are likely to be among those with the longest periods expected, this difference appears real. To try to understand the longest observed period, the synthetic AGB code of Wagenhuber \& Groenewegen (1998, A\&A, 340, 183) was fine-tuned to reproduce the models of Vassiliadis \& Wood (1993, ApJ, 413, 641). For several initial masses the fundamental mode period distribution was calculated for stars inside observed instability strip. Depending on details of the adopted mass loss rate, it is found that the mass limit where a carbon star has a probability of less than $1 \%$ of being in the observed instability strip with a period longer than 900 days is between 2.6 and $3.1 M_{\odot}$.

Conclusions. Synthetic AGB calculations suggest that the observed upper limit in period can be interpreted as an upper mass limit of carbon star formation, with a value of between 2.6 and 3.1 $M_{\odot}$, depending on the adopted AGB mass loss rate. Such a mass limit is predicted by stellar evolution through the occurence of Hot Bottom Burning where (dredged-up) carbon is converted into nitrogen; this is predicted to occur at higher masses $\left(\sim 4 M_{\odot}\right)$, although this depends on convection and core overshoot.
\end{abstract}

Key words. stars: carbon - stars: AGB and post-AGB - stars: variables: general - infrared: stars

\section{Introduction}

Pulsation is a critical aspect of the late stages of stellar evolution. The longest known indication of these pulsations are the well-expressed regular and irregular variations in brightness of objects on the Asymptotic Giant Branch (AGB) like Mira-, Semiregular (SRV), and Irregular Variables (IRV). This pulsation affects the structure of the stellar atmosphere. As it drives the extension of the outer stellar layers, it produces an environment for the formation of dust, which is an important factor for the mass loss and therefore for the stellar evolution.

The theoretical description of the pulsational phenomena in the atmosphere of AGB stars is now making considerable progress (e.g., Höfner et al. 1998; Höfner 1999; Winters et al. 2000; Nowotny et al. 2005, and references therein).

Moreover, for the more regular pulsators among the AGB variables, well established Mira period-luminosity and period- $K$ magnitude relations are available (e.g., Feast et al. 1989; Groenewegen \& Whitelock 1996), allowing for a reliable determination of distances to individual field stars or even outside the local group (e.g., the Miras in Cen A, Rejkuba 2004).

\footnotetext{
* Based on observations made with the Carlos Sánchez Telescope operated on the island of Tenerife by the Instituto de Astrofísica de Canarias (IAC) in the Observatorio del Teide, Izaña.

$\star \star$ Appendix A is only available in electronic form at http: //www . aanda.org
}

The results from the micro-lensing surveys are very impressive (e.g., Wood et al. 1999; Ita et al. 2004; Groenewegen 2004; Fraser et al. 2005) and have revealed several sequences that can be interpreted as being due to the fundamental mode pulsation of the Mira variables, and overtone pulsation for the smaller amplitude variables.

Also, robotic large area or even all sky surveys like the All Sky Automated Survey (ASAS, Pojmanski 2002) or the Northern Sky Variability Survey (NSVS, Wozniak et al. 2004) provide valuable and systematic information on AGB variability (see also Sect. 3.1).

Nevertheless, these surveys have been carried out in the optical and therefore miss the most obscured stars that undergo significant mass loss and then become faint to invisible in the optical. One should note that Kiss \& Bedding (2004) and Groenewegen (2004) did find a large number of very red $(J-K \gg 1.4 \mathrm{mag})$ "faint Miras" in the OGLE-II observations of the SMC, which fall below the fiducial Mira P-L relation by 1.5-2 mag in $K$ and 4-6 mag in $I$, so that they are very likely candidates for being obscured carbon-rich Mira stars.

The pulsational properties of such stars are poorly studied, in particular the carbon-rich ones, the so-called "infrared carbon stars". Few periods have been determined, except, e.g., by Jones et al. (1990) and Le Bertre (1992). Interestingly, the longest known period for a carbon star is around 780 days in the Galaxy (AFGL 809, AFGL 2494, Jones et al. 1990) and near 940 days in the LMC (IRAS 05190-6748, Whitelock et al. 2003), while 
among the dust obscured oxygen-rich stars (often called OH/IR stars), periods of well over a 1000 days and up to 2800 days are known (e.g., van Langevelde et al. 1990). Is this an evolutionary effect?

From observations in the LMC and SMC, it has become clear that the brightest AGB stars are not carbon stars, but MS and S-stars (e.g., Smith et al. 1995). This should be reflected in the periods carbon stars can attain. It is clear however that many more periods for infrared carbon stars are needed to quantify this.

Therefore, we present in this paper new multi-epoch nearinfrared photometry of a sample of IR bright, high mass loss carbon stars not studied in the optical range at all. For most of the objects in our sample this is the first measurement of their infrared light change.

\section{The sample and observations}

The sample was selected as follows: as a first step stars with an IRAS LRS classification of $4 \mathrm{n}$, suggestive of a carbon star as it indicates the presence of the silicon carbide dust feature (this was verified visually), and IRAS fluxes S25 >0.4 $\times$ S12 and S12 > 50 Jy were selected from the IRAS PSC. The former criterium selects carbon stars that, based on existing data, preferentially contain stars with $P>500$ days. The latter criterium ensures that even the reddest stars can be observed in $K$ and $L$ $(K<10)$, while the bluer stars can be monitored in JHKL. In addition, carbon stars with expansion velocities larger than about $23 \mathrm{~km} \mathrm{~s}^{-1}$ (selected from the catalogue of CO measurements of Loup et al. 1993) were added to the sample with the rationale that high expansion velocities are a sign of high luminosities in the theory of radiation driven wind (Elitzur \& Ivezić 2001) and carbon stars with high luminosities based on reasonably well determined kinematic distances (Kastner et al. 1993). Those additional stars that are outside the colour and/or flux criterium were only included if existing NIR data indicated that they are not too faint.

The observations were carried out at the Observatorio del Teide, the $1.5 \mathrm{~m}$ "Carlos Sánchez Telescope" of the Instituto de Astrofísica de Canarias (IAC) on Izaña, Tenerife was used with the "CVF Photometer-Spectrophotometer". The $L$-filter there is actually $L^{\prime}(3.78 \mu \mathrm{m})$.

All sources were searched for at their IRAS positions, mostly in filter $K$. A diaphragm of $15^{\prime \prime}$ was used; beam-switching was done in the East-West direction with a throw of generally $20^{\prime \prime}$. Standard and programme stars were observed at similar air mass ranges to avoid the nonlinear effects of atmospheric extinction in the infrared. The $J H K L^{\prime}$-photometry was calibrated on the Koornneef (1983a,b) system.

The observations were done by the authors during 25 dedicated runs between September 1995 and August 1999. A few additional measurements where carried out by Stefano Bagnulo and Mathias Schultheis.

\section{Results}

\subsection{Period finding}

As noted already in Lebzelter (1999), classical methods for deriving a period, like Fourier analysis (Sperl 1998), alone do not always provide satisfying results for light curves of AGB variables. We therefore combined it with visual inspection (maxima, minima) and least square fitting techniques (Sperl 1998).
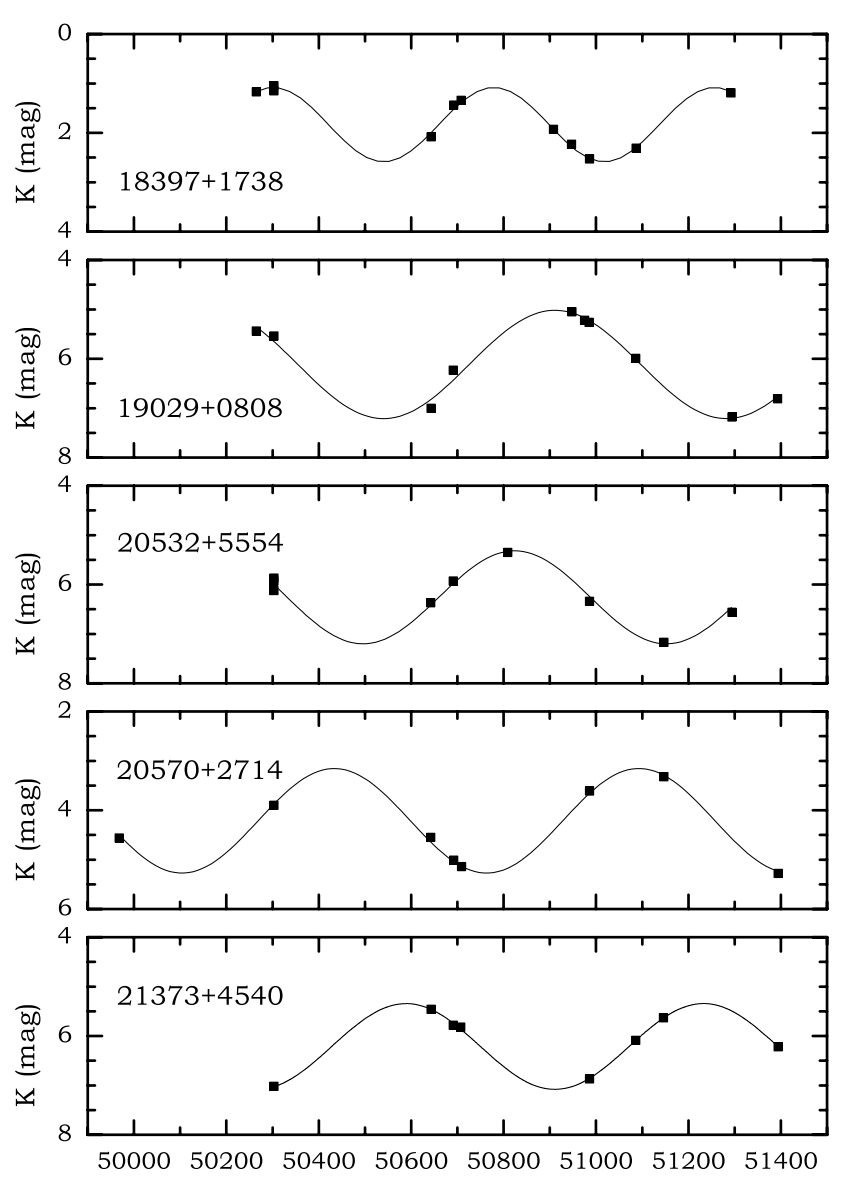

MJD (d)

Fig. 1. Examples of $K$-band photometry for 5 stars with corresponding fit solutions.

To illustrate the typical $K$-band variability of these stars we plotted the $K$-band light curves of five stars from our sample in Fig. 1. To allow a direct comparison of amplitude and period of the variation we used the same scaling for all stars shown.

The formal frequency uncertainty $\Delta f$ from Fourier analysis is indirectly proportional to the sample length $\Delta T$. A practical rule of thumb $\Delta f=1.5 / \Delta T$ comes from Loumos \& Deeming (1978), which corresponds, for our data sets ( $\Delta T$ typically 1000 days, which is of the same order as the expected periods) in the period domain, to $\Delta P$ of about 135,375 , and 735 days for periods of 300,500 , and 700 days, respectively. These very conservative values are considerably improved to about $10 \%$ of the period, when least-square fitting techniques are carried out, but one should keep in mind this principal limitation, which can only be overcome by a longer time base. Another limitation is the density of the sampling, which is responsible for the high frequency, i.e. short period cut-off. For our sampling (say 10 points in 1000 days, a typical Nyquist cut-off is found at around 200 days in period, below the expected periods of long period variables. A detection of more rapid variations or the detailed shape of the light curves would require a much better sampling.

Table 1 gives an overview of the resulting periods derived from the $K$-band light curves as well as the correponding amplitudes (used in the mathematical sense, i.e., half of the peak-topeak variation). Moreover, mean colours of the fitted light curves are calculated. Only in the two cases where no period solutions could be found were straight average colours calculated. $n$ gives 
Table 1. Mean near-infrared photometry, variability, luminosity and distance. $n$ gives the number of datapoints in the corresponding filter. A ":" after the $K$-band amplitude indicates uncertainties in period and amplitude determination. Luminosity and distance information is derived via the $P L$ relation discussed in the text.

\begin{tabular}{|c|c|c|c|c|c|c|c|c|c|c|c|c|c|c|c|c|c|}
\hline IRAS & Variable & Name & $\overline{J_{\mathrm{m}}}$ & $\overline{H_{\mathrm{m}}}$ & $\overline{K_{\mathrm{m}}}$ & $\overline{\overline{L_{\mathrm{m}}^{\prime}}}$ & $n_{\mathrm{J}}$ & $\overline{n_{\mathrm{H}}}$ & $\overline{n_{\mathrm{K}}}$ & $\bar{~} n_{\mathrm{L}^{\prime}}$ & 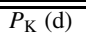 & $\overline{\overline{A_{\mathrm{K}}}}$ & & $\overline{M_{\mathrm{K}}}$ & $M_{\text {bol }}$ & 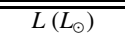 & $\overline{r_{\mathrm{K}}(\mathrm{pc})}$ \\
\hline $01105+6241$ & NSV 438 & AFGL177 & 5.27 & 3.63 & 2.35 & 0.56 & 7 & 7 & 7 & $\frac{2}{4}$ & 711 & 1.32 & $:$ & -9.01 & -5.37 & $1.08 \times 10^{4}$ & $1.87 \times 10^{3}$ \\
\hline $02152+2822$ & YY Tri & & 12.75 & 10.13 & 7.05 & 2.62 & 5 & 7 & 7 & 2 & 841 & 0.77 & & -9.27 & -5.55 & $1.29 \times 10^{4}$ & $1.84 \times 10^{4}$ \\
\hline $03192+5642$ & KX Cam & & 9.14 & 6.91 & 5.20 & 3.44 & 8 & 8 & 8 & 3 & 795 & 0.98 & : & -9.19 & -5.49 & $1.22 \times 10^{4}$ & $7.54 \times 10^{3}$ \\
\hline $03238+6034$ & KY Cam & AFGL4277 & 11.42 & 8.51 & 6.10 & 2.93 & 7 & 10 & 10 & 4 & 642 & 0.86 & & -8.86 & -5.25 & $9.75 \times 10^{3}$ & $9.81 \times 10^{3}$ \\
\hline $03385+5927$ & & & 12.32 & 9.61 & 6.92 & 3.42 & 6 & 10 & 10 & 2 & 629 & 1.02 & & -8.82 & -5.23 & $9.54 \times 10^{3}$ & $1.41 \times 10^{4}$ \\
\hline $03448+4432$ & & AFGL5102 & 13.69 & 10.47 & 7.24 & 2.99 & 6 & 8 & 8 & 3 & 729 & 0.73 & & -9.05 & -5.39 & $1.11 \times 10^{4}$ & $1.81 \times 10^{4}$ \\
\hline $04179+5951$ & & AFGL5118 & 10.76 & 8.03 & 5.76 & 2.90 & 3 & 7 & 7 & 1 & 536 & 0.88 & : & -8.58 & -5.05 & $8.08 \times 10^{3}$ & $7.36 \times 10^{3}$ \\
\hline $05136+4712$ & & & 11.65 & 9.14 & 6.85 & 3.46 & 6 & 9 & 9 & 4 & 552 & 0.85 & & -8.62 & -5.08 & $8.33 \times 10^{3}$ & $1.24 \times 10^{4}$ \\
\hline $06088+1909$ & & & 8.42 & 6.25 & 4.44 & 2.12 & 7 & 7 & 7 & 1 & 405 & 0.68 & & -8.14 & -4.73 & $6.04 \times 10^{3}$ & $3.28 \times 10^{3}$ \\
\hline $06226-0905$ & V636 Mon & AFGL933 & 4.96 & 3.32 & 1.99 & 0.50 & 5 & 5 & 5 & 3 & 490 & 0.78 & : & -8.44 & -4.95 & $7.37 \times 10^{3}$ & $1.22 \times 10^{3}$ \\
\hline $06268+0849$ & V477 Mon & AFGL5196 & 8.34 & 6.25 & 4.32 & 1.72 & 6 & 6 & 6 & 3 & 422 & 0.74 & : & -8.21 & -4.78 & $6.31 \times 10^{3}$ & $3.19 \times 10^{3}$ \\
\hline $06487+0551$ & & & 8.82 & 6.48 & 4.53 & 2.03 & 6 & 6 & 6 & 2 & 552 & 0.55 & : & -8.62 & -5.08 & $8.33 \times 10^{3}$ & $4.26 \times 10^{3}$ \\
\hline $06531-0216$ & & AFGL1039 & 6.93 & 5.12 & 3.62 & 1.83 & 6 & 6 & 6 & 1 & \multicolumn{3}{|c|}{ no solution } & & & & \\
\hline $17581-1744$ & & AFGL2047 & 8.07 & 5.89 & 4.12 & 1.82 & 10 & 10 & 10 & 5 & 590 & 0.71 & & -8.72 & -5.16 & $8.93 \times 10^{3}$ & $3.71 \times 10^{3}$ \\
\hline $18040-0941$ & FX Ser & AFGL 2067 & 6.56 & 4.21 & 2.45 & 0.35 & 8 & 8 & 8 & 4 & 605 & 0.67 & & -8.76 & -5.19 & $9.17 \times 10^{3}$ & $1.75 \times 10^{3}$ \\
\hline $18239-0655$ & NSV 24440 & AFGL2154 & 10.11 & 7.38 & 4.79 & 1.34 & 8 & 9 & 9 & 3 & 638 & 0.72 & : & -8.84 & -5.24 & $9.68 \times 10^{3}$ & $5.34 \times 10^{3}$ \\
\hline $18240+2326$ & V1076 Her & AFGL2155 & 11.76 & 9.29 & 6.02 & 1.42 & 7 & 8 & 8 & 5 & 662 & 0.93 & & -8.90 & -5.29 & $1.01 \times 10^{4}$ & $9.67 \times 10^{3}$ \\
\hline $18244-0815$ & & & 11.16 & 8.49 & 5.83 & 2.72 & 5 & 7 & 7 & 5 & 695 & 0.61 & & -8.98 & -5.34 & $1.06 \times 10^{4}$ & $9.13 \times 10^{3}$ \\
\hline $18248-0839$ & & & 12.66 & 9.42 & 6.34 & 2.25 & 6 & 6 & 8 & 4 & 652 & 1.01 & & -8.88 & -5.27 & $9.89 \times 10^{3}$ & $1.10 \times 10^{4}$ \\
\hline $18269-1257$ & & & 13.70 & 10.35 & 7.35 & 3.56 & 2 & 5 & 6 & 1 & 872 & 1.97 & & -9.33 & -5.60 & $1.34 \times 10^{4}$ & $2.16 \times 10^{4}$ \\
\hline $18320-0352$ & & AFGL7012 & 13.11 & 11.13 & 8.20 & 3.94 & 3 & 4 & 6 & 3 & 596 & 0.77 & : & -8.74 & -5.17 & $9.01 \times 10^{3}$ & $2.45 \times 10^{4}$ \\
\hline $18367-0452$ & & & 12.22 & 10.62 & 8.02 & 3.62 & 6 & 7 & 8 & 4 & 649 & 0.95 & & -8.87 & -5.26 & $9.85 \times 10^{3}$ & $2.39 \times 10^{4}$ \\
\hline $18397+1738$ & V821 Her & AFGL2232 & 5.56 & 3.60 & 1.83 & -0.74 & 11 & 11 & 11 & 7 & 478 & 0.75 & & -8.40 & -4.92 & $7.17 \times 10^{3}$ & $1.11 \times 10^{3}$ \\
\hline $18398-0220$ & V1417 Aql & AFGL2233 & 5.47 & 3.40 & 1.71 & -0.50 & 7 & 7 & 7 & 2 & 687 & 0.56 & & -8.96 & -5.33 & $1.04 \times 10^{4}$ & $1.36 \times 10^{3}$ \\
\hline $18424+0346$ & & & 9.42 & 6.95 & 4.91 & 2.26 & 8 & 8 & 8 & 2 & 456 & 1.07 & & -8.33 & -4.87 & $6.83 \times 10^{3}$ & $4.43 \times 10^{3}$ \\
\hline $18475+0926$ & & AFGL2259 & 11.58 & 9.20 & 6.41 & 2.22 & 8 & 10 & 10 & 4 & 610 & 0.80 & & -8.78 & -5.19 & $9.24 \times 10^{3}$ & $1.09 \times 10^{4}$ \\
\hline $19029+0808$ & NSV 24681 & AFGL2316 & 11.45 & 9.08 & 6.11 & 2.05 & 7 & 10 & 10 & 4 & 742 & 1.10 & & -9.08 & -5.41 & $1.13 \times 10^{4}$ & $1.09 \times 10^{4}$ \\
\hline $19029+2017$ & & AFGL2318 & 7.83 & 5.65 & 3.86 & 1.84 & 6 & 6 & 6 & 3 & 594 & 0.68 & & -8.73 & -5.16 & $8.99 \times 10^{3}$ & $3.30 \times 10^{3}$ \\
\hline $19068+0544$ & & & 7.84 & 5.57 & 3.94 & 2.02 & 8 & 8 & 8 & 2 & 661 & 0.85 & & -8.90 & -5.28 & $1.00 \times 10^{4}$ & $3.69 \times 10^{3}$ \\
\hline $19175-0807$ & V1420 Aql & & 7.22 & 4.71 & 2.66 & -0.15 & 10 & 10 & 10 & 5 & 636 & 1.10 & & -8.84 & -5.24 & $9.65 \times 10^{3}$ & $2.00 \times 10^{3}$ \\
\hline $19455+0920$ & & AFGL4253 & 11.93 & 9.34 & 6.68 & 3.01 & 7 & 6 & 7 & 2 & 538 & 0.99 & & -8.58 & -5.05 & $8.11 \times 10^{3}$ & $1.13 \times 10^{4}$ \\
\hline $19548+3035$ & & AFGL2477 & 10.15 & 8.94 & 8.46 & 6.57 & 4 & 5 & 5 & 2 & \multicolumn{2}{|c|}{ no solution } & & & & & \\
\hline $20072+3116$ & V1969 Cyg & AFGL2513 & 7.54 & 5.30 & 3.43 & 0.95 & 9 & 9 & 9 & 4 & 706 & 0.96 & & -9.00 & -5.36 & $1.07 \times 10^{4}$ & $3.07 \times 10^{3}$ \\
\hline $20082+3228$ & & & 11.56 & 8.68 & 6.14 & 2.82 & 5 & 6 & 6 & 2 & 654 & 0.91 & & -8.88 & -5.27 & $9.93 \times 10^{3}$ & $1.01 \times 10^{4}$ \\
\hline $20171+3519$ & & & 11.72 & 10.91 & 8.04 & 3.74 & 3 & 4 & 5 & 2 & 860 & 0.94 & : & -9.31 & -5.58 & $1.32 \times 10^{4}$ & $2.94 \times 10^{4}$ \\
\hline $20435+3825$ & & & 11.46 & 8.45 & 5.84 & 2.17 & 8 & 9 & 9 & 4 & 487 & 0.49 & : & -8.43 & -4.94 & $7.32 \times 10^{3}$ & $7.13 \times 10^{3}$ \\
\hline $20532+5554$ & V703 Cep & & 11.95 & 8.99 & 6.26 & 2.49 & 5 & 9 & 9 & 2 & 656 & 0.94 & & -8.89 & -5.28 & $9.97 \times 10^{3}$ & $1.07 \times 10^{4}$ \\
\hline $20570+2714$ & V442 Vul & AFGL2686 & 9.49 & 6.83 & 4.21 & 0.63 & 9 & 8 & 8 & 5 & 659 & 1.06 & & -8.90 & -5.28 & $1.00 \times 10^{4}$ & $4.19 \times 10^{3}$ \\
\hline $21003+4801$ & & & 10.54 & 7.84 & 5.35 & 2.08 & 6 & 7 & 7 & 4 & 766 & 0.55 & & -9.13 & -5.45 & $1.17 \times 10^{4}$ & $7.87 \times 10^{3}$ \\
\hline $21027+5309$ & V1899 Cyg & AFGL2699 & 9.71 & 7.33 & 5.15 & 2.29 & 8 & 8 & 8 & 5 & 662 & 0.69 & & -8.90 & -5.29 & $1.00 \times 10^{4}$ & $6.47 \times 10^{3}$ \\
\hline $21147+5110$ & & & 13.18 & 11.34 & 8.40 & 3.86 & 6 & 7 & 8 & 4 & 772 & 0.99 & & -9.14 & -5.46 & $1.18 \times 10^{4}$ & $3.21 \times 10^{4}$ \\
\hline $21223+5114$ & & & 13.35 & 10.05 & 7.14 & 3.40 & 4 & 5 & 6 & 2 & 663 & 0.88 & & -8.91 & -5.29 & $1.01 \times 10^{4}$ & $1.62 \times 10^{4}$ \\
\hline $21373+4540$ & & & 11.35 & 8.69 & 6.21 & 2.78 & 5 & 8 & 8 & 3 & 644 & 0.87 & & -8.86 & -5.25 & $9.77 \times 10^{3}$ & $1.03 \times 10^{4}$ \\
\hline $21449+4950$ & V2358 Cyg & & 11.43 & 8.65 & 6.24 & 3.13 & 6 & 8 & 8 & 2 & 665 & 0.60 & : & -8.91 & -5.29 & $1.01 \times 10^{4}$ & $1.07 \times 10^{4}$ \\
\hline $22241+6005$ & V384 Cep & AFGL2901 & 9.60 & 6.91 & 4.42 & 1.19 & 8 & 9 & 9 & 4 & 641 & 0.74 & : & -8.85 & -5.25 & $9.73 \times 10^{3}$ & $4.51 \times 10^{3}$ \\
\hline $23174+5941$ & V571 Cas & & 9.43 & 7.16 & 5.22 & 2.73 & 9 & 9 & 9 & 4 & 509 & 0.63 & : & -8.50 & -4.99 & $7.66 \times 10^{3}$ & $5.54 \times 10^{3}$ \\
\hline $23279+5336$ & & & 9.86 & 7.54 & 5.40 & 2.85 & 7 & 6 & 8 & 5 & 583 & 0.69 & $:$ & -8.71 & -5.14 & $8.81 \times 10^{3}$ & $6.64 \times 10^{3}$ \\
\hline
\end{tabular}

the number of datapoints in the corresponding filter. A ":" after the $K$-band amplitude indicates uncertainties in period and amplitude determination mostly because of poor sampling of the light curves.

To visualise our attempts to fit the observed light curves, Fig. 1 gives five examples. It is obvious that the phase coverage of our NIR measurements is not comparable with well-sampled light curves in the visual. Sometimes it is better to speak of multi-epoch photometry. Nevertheless it can be used to estimate a better average luminosity or at least to get a lower limit for the pulsational amplitudes in the infrared.

Only a few objects of our sample have already published periods. In total, 13 stars were also independently monitored by Whitelock et al. (2006). For two C stars we came to exactly (within a few days) the same periods, namely, IRAS $18239-0655$ and $20570+2714$, which is quite a surprise given the intrinsic uncertainties in period determination as discussed above. Seven additional objects only deviate up to about $10 \%$ in their periods. Using our new periods the datasets of IRAS 06226-0905, 06487+0551, 17581-1744, 18240+2326, 18397+1738 (see Fig. 1), 18398-0220, and 19175-0807 can typically be fitted with residuals of a half or a third of those with the published periods. Nevertheless, taking into account the very small number of points in the individual light curves, this is not a final proof. For only four stars we arrived at different periods. In the case of IRAS $02152+2822$ we found 841 instead of 624 days; for $06088+1909,405$ instead of 493 days; for $06268+0849,422$ instead of 619 days, and for 18040-0941, 605 instead of 519 days. In all cases we tried the published values again but even after wider variations no fit solutions with comparable small residuals could be found. Again, the limited datasets leave some uncertainties since the results could be different with only a few points added or deleted.

A quite similar comparison was carried out for the few objects that have positional counterparts (variables within one arcminute) in the NSVS (see above, for ASAS no counterparts were found). In the case of NSVS six stars match the J2000 IRAS coordinates within only 5 arcsec. Three NSVS objects, corresponding to IRAS $03192+5642,19029+2017$ and $19068+0544$ support our period findings very well.

The three others, IRAS $01105+6241,18040-0941$, and $18397+1738$, all show very well defined but quite different variability in their NSVS data sets, in the 250 to 650 day period ranges with amplitudes well below $1 \mathrm{mag}$. All are clearly 


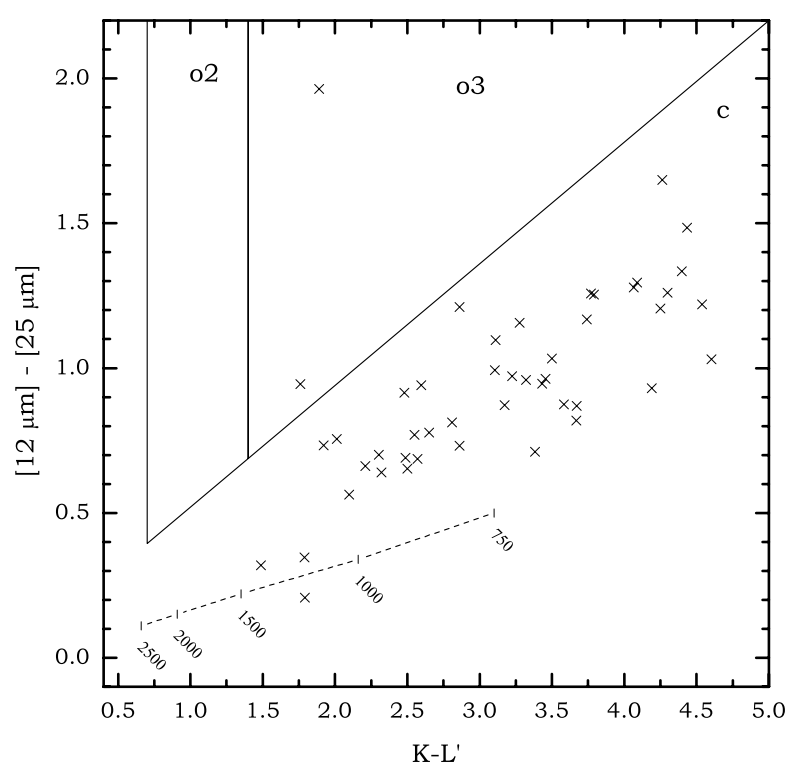

Fig. 2. Infrared two colour diagram according to that of Epchtein et al. (1987) to discriminate O-rich from carbon stars. The colours for blackbodies of different temperatures $[\mathrm{K}]$ are indicated. The two outliers in box " 03 " are discussed in the text.

incompatible with our IR material. The most probable interpretation is in terms of an incidental positional association.

\subsection{Mean colours}

Using the light curve fits described above, it is possible to derive mean magnitudes in all filters. For some objects with very few measurements in certain colours (mainly $L_{\mathrm{m}}^{\prime}$ ), no fit was possible and the mean colour index between simultaneous measurements in $K$ and the respective other filter " $\mathrm{X}$ " was used to calculate the colour index between the mean $K$ magnitude $K_{\mathrm{m}}$ and " $X_{\mathrm{m}}$ ".

Taking our mean colour indices and corresponding IRAS fluxes ${ }^{1}$ from the IRAS point source catalogue (PSC, 1988), the infrared two colour diagram shown in Fig. 2 was produced. Following Epchtein et al. (1987), it can be used to characterise AGB objects according to their mass loss rates and chemical properties. Regions "o2" and "o3" contain mainly O-rich stars with increasing mass loss towards higher $\left(K-L^{\prime}\right)$ indices. To the left of them, "pure" photosphere without mass loss would be situated. Below in the large area denoted "c", towards the blackbody line, carbon stars of different mass loss rates can be found. Again, higher rates are found to the right.

The aim of the original selection described in Sect. 2 was to select carbon stars. The data points in Fig. 2 confirm the carbon star nature for the overall majority of the stars.

The star located at $(K-L)=1.76$ and $[12 \mu \mathrm{m}]-[25 \mu \mathrm{m}]=0.95$ is IRAS $03192+5642$. It is located quite close to the borderline between regions "o3" and "c". This is unquestionably a carbon star because of its strong $\mathrm{SiC}$ feature and its listing in the "General catalog of galactic carbon stars" (Alksnis et al. 2001).

The object located far outside region "c" in the upper part of Fig. 2 is IRAS $19548+3035$ (AFGL 2477). This object was

1 The use of IRAS-PSC magnitudes varies in the literature. Both zero point corrected and not zero point corrected values are found. To avoid confusion, for this paper we defined a zero point corrected $[x \mu \mathrm{m}] \equiv$ $-2.5 \cdot \log \left(F / F_{0}\right)_{x \mu \mathrm{m}}$. The $F_{0}$-values were taken from the IRAS-PSC.

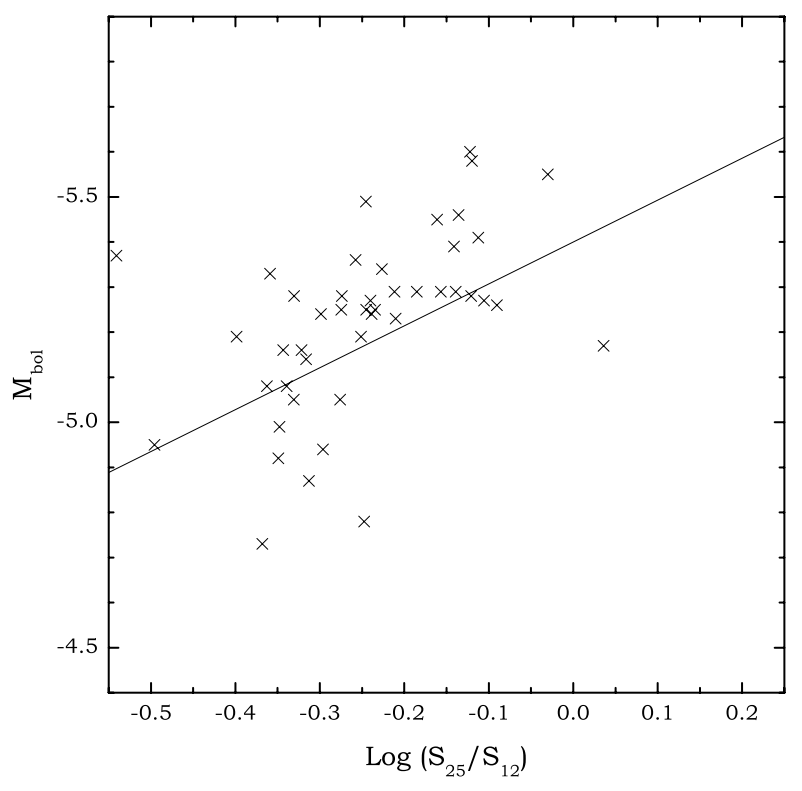

Fig. 3. Bolometric magnitude and IRAS flux-ratio. The line indicates the relation given in Groenewegen et al. (2002).

previously studied by Groenewegen et al. (1996). This is a very interesting star with a bi-modal spectral energy distribution with a M6S star dominating the light from the optical up to $\sim 3 \mu \mathrm{m}$ and optically thick carbon-rich material (as evidenced from the IRAS LRS spectrum and molecular line emission) emitting longwards. The JHK magnitudes trace the M6S star and our observations then imply that this star is not variable. Our magnitudes are in good agreement with 2MASS $(J=10.20, H=8.98, K=8.46)$. Our new observations do not resolve the nature of this object, which could be a chance coincidence on the sky, or a peculiar binary system with two AGB stars possibly in a disk configuration (Groenewegen et al. 1996).

\subsection{Absolute magnitudes and distances}

Using the period-luminosity and period- $K$ magnitude relations given in Groenewegen \& Whitelock (1996), absolute $K$ magnitudes $M_{\mathrm{K}}$, bolometric magnitudes $M_{\mathrm{bol}}$, as well as luminosities $L$ were calculated and listed in Table 1 . Together with the mean apparent $K$ magnitudes individual distances $r_{\mathrm{K}}$ could be derived and are also listed there.

In Groenewegen et al. (2002), a relation between bolometric magnitudes and an IRAS flux ratio was established to derive distances. Our independent dataset can be used to check this relation. The corresponding plot is shown in Fig. 3. For reasons discussed above, IRAS $19548+3035$ was omitted in the figure.

\section{Discussion and conclusions}

Near-infrared observations for a sample of 47 infrared carbon stars are presented, with reliable periods for 31 of them. All amplitudes are such that these objects are Mira type variables. The longest two periods are 840 and 870 days, only slightly longer than the previously longest known period for a galactic carbon star of 783 days. Still, this is considerably shorter than the periods of some $\mathrm{OH} / \mathrm{IR}$ stars. As the present survey was targeted at carbon stars that are likely to be among those with the longest periods expected, this difference appears real. 

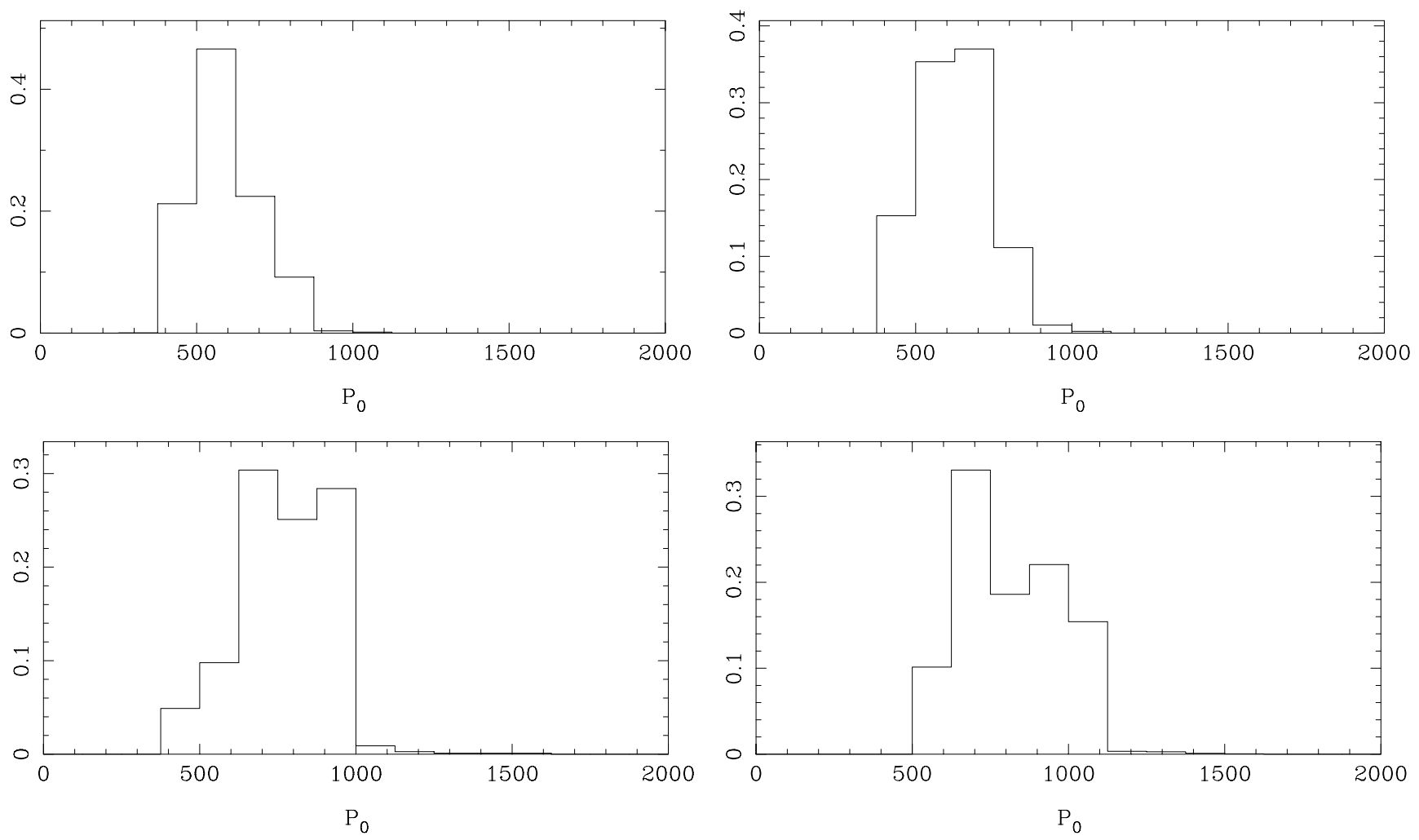

Fig. 4. Theoretical period distribution of AGB stars inside the observed instability strip for carbon stars for masses of 2.5, 2.6, 3.0 (all at $Z=0.016$ ), and 3.0 (at $Z=0.010) M_{\odot}$ (left to right, top to bottom). Every histogram is normalised to unity.

To try to quantify the relation between pulsation period and stellar evolution, synthetic AGB evolutionary models have been calculated, very similar to the ones described in detail in Appendix C of Groenewegen \& Blommaert (2005).

In brief, the synthetic AGB code of Wagenhuber \& Groenewegen (1998) was fine-tuned to reproduce the models of Vassiliadis \& Wood (1993; hereafter VW) and then extended to more initial masses, including mass loss on the RGB. For several initial masses the fundamental mode period distribution was calculated for stars inside observed instability strips. Contrary to the calculations presented in Groenewegen \& Blommaert (2005) where only the optically visible phase was considered, the present work considers the entire LPV phase.

In the present work two $P L$ relations are considered, that of Feast et al. (1989) for O-rich stars,

$M_{\text {bol }}=-3.00 \log P+2.85$,

and that of Groenewegen \& Whitelock (1996) for C stars,

$M_{\mathrm{bol}}=-2.59 \log P+2.02$.

For each timestep in the synthetic AGB calculation the fundamental period is calculated following VW. The star is assumed to be in the Mira instability strip when the bolometric magnitude is within 0.20 magnitude (the assumed width of the instability strip at a given period) of the $P L$ relation.

Table 2 lists the AGB lifetime (defined as the time between the first model in the file (the start of the AGB) up to the point where the remaining envelope mass becomes less than $0.04 M_{\odot}$, or $T_{\text {eff }}>4500 \mathrm{~K}$, that is taken as the start of the post-AGB evolution) for several initial masses, and the LPV lifetime for both $P L$ relations considered.

Figures 4 and 5 display the distribution of the fundamental period considering the $P L$ relation for C- and O-rich stars,
Table 2. AGB and LPV lifetimes.

\begin{tabular}{rrrrr}
\hline \hline$Z$ & Mass & AGB lifetime & \multicolumn{2}{c}{ LPV lifetime } \\
& & & $\mathrm{O}$ & $\mathrm{C}$ \\
& $\left(M_{\odot}\right)$ & $\left(10^{3}\right.$ years $)$ & $\left(10^{3}\right.$ years $)$ & $\left(10^{3}\right.$ years $)$ \\
\hline 0.016 & 2.5 & 2269 & 101 & 49 \\
0.016 & 2.6 & 2301 & 70 & 43 \\
0.016 & 3.0 & 1643 & 36 & 35 \\
0.016 & 3.5 & 497 & 25 & 26 \\
0.016 & 4.0 & 232 & 18 & 21 \\
0.016 & 4.5 & 191 & 16 & 19 \\
0.016 & 5.0 & 175 & 15 & 14 \\
0.010 & 2.5 & 2088 & 78 & 45 \\
0.010 & 2.6 & 2092 & 59 & 45 \\
0.010 & 3.0 & 1434 & 32 & 33 \\
0.016 & 2.8 & $2141^{1}$ & $207^{1}$ & $257^{1}$ \\
0.016 & 3.1 & $894^{2}$ & $107^{2}$ & $133^{2}$ \\
\hline
\end{tabular}

${ }^{1}$ For a Reimers mass loss with scaling factor $1.7 .{ }^{2}$ For a Reimers mass loss with scaling factor 4.0.

respectively. For the O-rich stars, periods of $\gtrsim 1500$ days are reached for the most massive star considered, in agreement with observations. The calculations predict that for a $2.5 M_{\odot}$ the fraction of time spent in the $\mathrm{C}$ star $P L$ relation at periods of $\gtrsim 900$ days is only $0.1 \%$, for $2.6 M_{\odot}$ stars this is $1 \%$, while for $3.0 M_{\odot}$ stars it is already $\sim 30 \%$. A model for a $3 M_{\odot}$ star at a lower metallicity is presented and this object spends more time at the longest periods. These calculations qualitatively reproduce the observations that the longest known period is slightly longer in the LMC than in the Galaxy. The calculations also suggest that the upper mass limit for $\mathrm{C}$ star formation is near $2.6 M_{\odot}$.

This "critical" initial mass limit does depend on the adopted mass loss rate. The last two entries of Table 2 are for a Reimers 

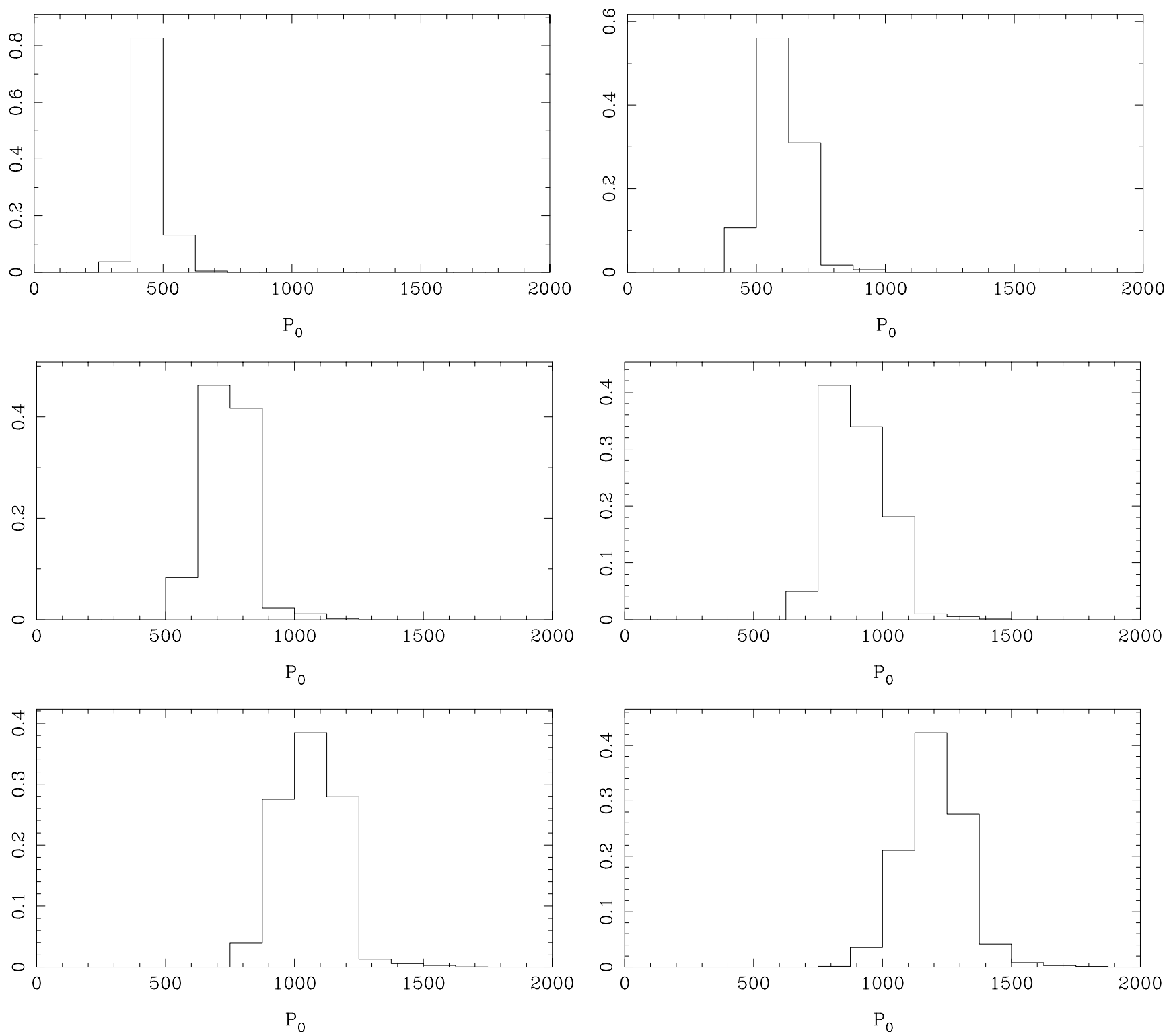

Fig. 5. Theoretical period distribution of AGB stars inside the observed instability strip for oxygen-rich stars for masses of 2.5, 3.0, 3.5, 4.0, 4.5, and $5.0 M_{\odot}$ (left to right, top to bottom). Every histogram is normalised to unity.

mass loss rate. One for a scaling factor of 1.7, which was finetuned to give the same AGB lifetime as the VW model for a $2.5 M_{\odot}$ star. In that case the initial mass that gives a $1 \%$ probability of having a period of $\gtrsim 900$ days inside the $\mathrm{C}$ star stability strip is raised to $2.8 M_{\odot}$. The last model is for a Reimers scaling factor of 4.0, which is the preferred value from Groenewegen et al. (1995), based on several considerations. In that case the "critical" initial mass is $3.1 M_{\odot}$.

The main argument for an upper limit in initial mass for C star formation is the process of Hot Bottom Burning (HBB) where carbon is converted to nitrogen. The main confirmation of this is the fact that almost all of the brightest AGB stars in the LMC are oxygen-rich and lithium-rich (e.g., Smith et al. 1995). As an aside it is noted that at the very end of the AGB the envelope mass drops so low that HBB stops, and the star may again become a $\mathrm{C}$ star if a third dredge-up occurs again. This implies that in a rare case a carbon star may appear as a Mira variable, probably at a period much longer than 900 days.

Theoretical predictions by Mazzitelli et al. (1999) and Ventura et al. (2000) indicate that HBB occurs for initial masses $\gtrsim 4 M_{\odot}$, but this depends on the level of core overshoot and convective mixing, and mass loss. This mass limit is higher than that derived from the longest period of carbon stars. It is unclear how to resolve this discrepancy. Probably only dedicated stellar evolutionary calculations including dredge-up and considering various mass loss rates and input physics can show if the longest period for $\mathrm{C}$ stars can be linked directly to the onset of $\mathrm{HBB}$, and at which initial mass.

Acknowledgements. The work of F.K. was supported by APART (Austrian Programme for Advanced Research and Technology) from the Austrian Academy of Sciences.

\section{References}

Alksnis, A., Balklavs, A., Dzervitis, U., et al. 2001, BaltA, 10, 1 Elitzur, M., \& Ivezić, Ž. 2001, MNRAS, 327, 403

Epchtein, N., Le Bertre, T., Lepine, J. R. D., et al. 1987, A\&AS, 71, 39 Feast, M. W., Glass, I. S., Whitelock, P. A., \& Catchpole, R. M. 1989, MNRAS, 241,375

Fraser, O. J., Hawley, S. L., Cook, K. H., \& Keller, S. C. 2005, AJ, 129, 768

Groenewegen, M. A. T. 2004, A\&A, 425, 595 
Groenewegen, M. A. T., \& Blommaert, J. A. D. L. 2005, A\&A, 443, 143

Groenewegen, M. A. T., \& Whitelock, P. 1996, MNRAS, 281, 1347

Groenewegen, M. A. T., Oudmaijer, R. D., Goudfrooij, P., van den Hoek, L. B., \& van Kerkwijk, M. H. 1996, A\&A, 305, 475

Groenewegen, M. A. T., Van den Hoek, L. B., \& de Jong, T. 1995, A\&A, 293, 381

Groenewegen, M. A. T., Sevenster, M., Spoon, H. W. W., \& Pèrez, I. 2002, A\&A, 390, 501

Höfner, S. 1999, A\&A, 346, L9

Höfner, S., Jørgensen, U. G., Loidl, R., \& Aringer, B. 1998, A\&A, 340, 497

IRAS Science Team 1988, IRAS Catalogs and Atlases, Volumes 2-6, NASA RP-1190

Ita, Y., Tanabé, T., Matsunaga, N., et al. 2004, MNRAS, 347, 720

Jones, T. J., Bryja, C. O., Gehrz, T. E. H., et al. 1990, ApJS, 74, 785

Kastner, J. H., Forveille, T., Zuckerman, B., \& Omont, A. 1993, A\&A, 275, 163

Kiss, L., \& Bedding, T. 2004, MNRAS, 347, L83

Koornneef, J. 1983a, A\&AS, 51, 489

Koornneef, J. 1983b, A\&A, 128, 84

Le Bertre, T. 1992, A\&AS, 94, 377

Lebzelter, T. 1999, A\&A, 351, 644

Loumos, G. L., \& Deeming, T. J. 1978, Ap\&SS, 56, 285
Loup, C., Forveille, T., Omont, A., \& Paul, J. F. 1993, A\&AS, 99, 291

Mazzitelli, I., D’Antona, F., \& Ventura, P. 1999, A\&A, 348, 846

Nowotny, W., Lebzelter, T., Hron, J., \& Höfner, S. 2005, A\&A, 437, 285

Pojmansky, G. 2002, Acta Astronomica, 52, 397

Rejkuba M. 2004, A\&A, 413, 903

Smith, V. V., Plez, B., Lambert, D. L., \& Lubowich, D. A. 1995, ApJ, 441, 735

Sperl, M. 1998, Comm. Asteroseismology (Vienna), 111, 1

van Langevelde, H. J., van der Heiden, R., \& van Schooneveld, C. 1990, A\&A, 239,193

Vassiliadis, E, \& Wood, P. R. 1993, ApJ, 413, 641 (VW)

Ventura, P., Mazzitelli, I., \& D’Antona, F. 2000, A\&A, 363, 605

Wagenhuber, J., \& Groenewegen, M. A. T. 1998, A\&A, 340, 183

Whitelock, P. A., Feast, M. W., van Loon, J. Th., \& Zijlstra, A. A. 2003, MNRAS, 342, 86

Whitelock, P. A., Feast, M. W., Marang, F., \& Groenewegen, M. A. T. 2006, MNRAS, 369, 751

Winters, J. M., Keady, J. J., Gauger, A., \& Sada, P. V. 2000, A\&A, 359, 651

Wood, P. R., Alcock, C., Allsman, R. A., et al. 1999, in Asymptotic Giant Branch Stars, ed. T. Le Bertre, A. Lèbre, \& C. Waelkens, IAU Symp., 191, ASP, 151

Wozniak, P. R., Vestrand, W. T., Akerlof, C. W., et al. 2004, AJ, 127, 2436 
F. Kerschbaum et al.: Near-infrared variability of a sample of galactic carbon Miras, Online Material $p 1$

\section{Online Material}


F. Kerschbaum et al.: Near-infrared variability of a sample of galactic carbon Miras, Online Material p 2

Table A.1. Mean total errors of NIR photometry.

\begin{tabular}{ccccc}
\hline \hline Mag & $\sigma_{\mathrm{J}}$ & $\sigma_{\mathrm{H}}$ & $\sigma_{\mathrm{K}}$ & $\sigma_{\mathrm{L}^{\prime}}$ \\
\hline$<5$ & $0^{\mathrm{m}} 02$ & $0^{\mathrm{m}} 02$ & $0^{\mathrm{m}} 02$ & 0.02 \\
$5-8$ & 0.03 & 0.02 & 0.02 & 0.04 \\
$8-11$ & 0.04 & 0.03 & 0.03 & \\
$11-14$ & 0.06 & & & \\
\hline
\end{tabular}

\section{Appendix A: NIR photometry}

Estimates of the mean total errors of the NIR photometry are given in Table A.1. The given values are calculated from the individual programme- and standard-star measurements. The errors do not include uncertainties of the photometric system.

Table A.2 lists all near-infrared photometry presented in this paper. Besides IRAS name, the modified Julian day number is given. 
F. Kerschbaum et al.: Near-infrared variability of a sample of galactic carbon Miras, Online Material p 3

Table A.2. Individual NIR photometry.

\begin{tabular}{|c|c|c|c|c|c|}
\hline$\overline{\overline{\text { IRAS }}}$ & $\overline{\overline{\text { MJD }}}$ & $\overline{\bar{J}}$ & $\overline{\bar{H}}$ & $\overline{\bar{K}}$ & $\overline{\overline{L^{\prime}}}$ \\
\hline $01105+6241$ & 50407.0 & 4.97 & 3.24 & 1.90 & 0.27 \\
\hline $01105+6241$ & 50498.0 & 4.34 & 2.69 & 1.42 & \\
\hline $01105+6241$ & 50644.0 & 4.58 & 2.86 & 1.54 & -0.11 \\
\hline $01105+6241$ & 50690.0 & 4.91 & 3.16 & 1.78 & \\
\hline $01105+6241$ & 50707.0 & 5.06 & 3.30 & 1.91 & 0.23 \\
\hline $01105+6241$ & 51085.1 & 5.15 & 3.78 & 2.77 & 0.56 \\
\hline $01105+6241$ & 51144.9 & 4.13 & 2.58 & 1.34 & \\
\hline $02152+2822$ & 50303.0 & & 9.40 & 6.31 & \\
\hline $02152+2822$ & 50303.0 & & 9.35 & 6.30 & \\
\hline $02152+2822$ & 50407.0 & 12.11 & 9.31 & 6.31 & 2.05 \\
\hline $02152+2822$ & 50692.0 & 13.30 & 10.93 & 7.81 & \\
\hline $02152+2822$ & 50708.0 & 13.30 & 10.94 & 7.83 & 3.22 \\
\hline $02152+2822$ & 50809.0 & 13.10 & 10.76 & 7.65 & \\
\hline $02152+2822$ & 51086.1 & 12.80 & 9.54 & 6.52 & \\
\hline $03192+5642$ & 50303.0 & 9.31 & 7.01 & 5.27 & \\
\hline $03192+5642$ & 50303.0 & 9.19 & 6.93 & 5.21 & \\
\hline $03192+5642$ & 50408.0 & 8.06 & 6.01 & 4.50 & 2.51 \\
\hline $03192+5642$ & 50690.0 & 9.19 & 6.90 & 5.18 & \\
\hline $03192+5642$ & 50692.0 & 9.13 & 6.85 & 5.14 & \\
\hline $03192+5642$ & 50707.0 & 8.98 & 6.74 & 5.08 & 3.23 \\
\hline $03192+5642$ & 51085.2 & 9.59 & 7.33 & 5.37 & 3.93 \\
\hline $03192+5642$ & 51144.9 & 8.68 & 6.47 & 4.85 & \\
\hline $03238+6034$ & 50303.0 & & 9.32 & 6.82 & \\
\hline $03238+6034$ & 50303.0 & & 9.20 & 6.82 & \\
\hline $03238+6034$ & 50408.0 & 11.90 & 8.84 & 6.47 & 3.16 \\
\hline $03238+6034$ & 50498.0 & & 7.81 & 5.56 & \\
\hline $03238+6034$ & 50692.0 & 10.86 & 7.96 & 5.61 & \\
\hline $03238+6034$ & 50709.0 & 11.20 & 8.12 & 5.74 & 2.57 \\
\hline $03238+6034$ & 50809.0 & 12.20 & 9.34 & 6.73 & \\
\hline $03238+6034$ & 50877.0 & 12.40 & 9.35 & 6.77 & 3.30 \\
\hline $03238+6034$ & 51085.2 & 11.40 & 8.77 & 6.41 & 3.67 \\
\hline $03238+6034$ & 51144.9 & 10.20 & 7.51 & 5.21 & \\
\hline $03385+5927$ & 50303.0 & & 8.90 & 6.32 & \\
\hline $03385+5927$ & 50303.0 & & 8.76 & 6.20 & \\
\hline $03385+5927$ & 50408.0 & 11.26 & 8.34 & 5.81 & 2.42 \\
\hline $03385+5927$ & 50498.0 & & 9.14 & 6.51 & \\
\hline $03385+5927$ & 50690.0 & & 10.57 & 7.79 & \\
\hline $03385+5927$ & 50692.0 & 12.80 & 10.64 & 7.83 & \\
\hline $03385+5927$ & 50811.0 & 12.60 & 10.67 & 7.73 & \\
\hline $03385+5927$ & 50877.0 & 12.90 & 9.44 & 6.70 & \\
\hline $03385+5927$ & 51087.1 & 11.60 & 8.73 & 6.09 & 2.48 \\
\hline $03385+5927$ & 51145.0 & 12.10 & 9.43 & 6.68 & \\
\hline $03448+4432$ & 49970.0 & 14.90 & 11.49 & 8.20 & 3.80 \\
\hline $03448+4432$ & 50303.0 & & 9.60 & 6.56 & \\
\hline $03448+4432$ & 50407.0 & 12.66 & 9.70 & 6.72 & 2.58 \\
\hline $03448+4432$ & 50690.0 & & 11.42 & 7.87 & \\
\hline $03448+4432$ & 50692.0 & 14.30 & 11.17 & 7.91 & \\
\hline $03448+4432$ & 50708.0 & 15.90 & 11.14 & 7.91 & 3.70 \\
\hline $03448+4432$ & 50809.0 & 13.50 & 10.96 & 7.57 & \\
\hline $03448+4432$ & 51145.0 & 12.40 & 9.74 & 6.68 & \\
\hline $04179+5951$ & 50303.0 & & 8.20 & 5.94 & \\
\hline $04179+5951$ & 50303.0 & & 8.26 & 6.00 & \\
\hline $04179+5951$ & 50498.0 & & 8.30 & 6.04 & \\
\hline $04179+5951$ & 50691.0 & 10.04 & 7.45 & 4.93 & \\
\hline $04179+5951$ & 50809.0 & 11.15 & 8.39 & 5.94 & \\
\hline $04179+5951$ & 51085.2 & & 8.06 & 6.06 & 3.20 \\
\hline $04179+5951$ & 51145.0 & 9.52 & 7.01 & 4.85 & \\
\hline $05136+4712$ & 50303.0 & & 9.75 & 7.23 & \\
\hline $05136+4712$ & 50407.0 & 10.85 & 8.40 & 6.22 & 2.92 \\
\hline $05136+4712$ & 50497.0 & & 8.33 & 6.11 & \\
\hline
\end{tabular}


F. Kerschbaum et al.: Near-infrared variability of a sample of galactic carbon Miras, Online Material p 4

Table A.2. continued.

\begin{tabular}{|c|c|c|c|c|c|}
\hline$\overline{\text { IRAS }}$ & MJD & $\bar{J}$ & $\bar{H}$ & $\bar{K}$ & $\overline{L^{\prime}}$ \\
\hline $05136+4712$ & 50690.0 & & 9.80 & 7.45 & \\
\hline $05136+4712$ & 50692.0 & 12.30 & 9.77 & 7.47 & \\
\hline $05136+4712$ & 50709.0 & 12.70 & 9.86 & 7.55 & 4.08 \\
\hline $05136+4712$ & 50877.0 & 11.70 & 9.34 & 7.05 & 3.60 \\
\hline $05136+4712$ & 51087.2 & 10.80 & 8.21 & 6.00 & 2.69 \\
\hline $05136+4712$ & 51147.1 & 11.50 & 8.99 & 6.74 & \\
\hline $06088+1909$ & 50407.0 & 8.81 & 6.60 & 4.72 & 2.40 \\
\hline $06088+1909$ & 50498.0 & 9.26 & 7.04 & 5.19 & \\
\hline $06088+1909$ & 50690.0 & 7.65 & 5.60 & 3.84 & \\
\hline $06088+1909$ & 50809.0 & 8.92 & 6.70 & 4.89 & \\
\hline $06088+1909$ & 50811.0 & 8.92 & 6.73 & 4.96 & \\
\hline $06088+1909$ & 50877.0 & 9.00 & 6.79 & 4.89 & \\
\hline $06088+1909$ & 51145.1 & 7.72 & 5.66 & 3.94 & \\
\hline 06226-0905 & 50407.0 & 3.96 & 2.55 & 1.45 & 0.04 \\
\hline 06226-0905 & 50690.0 & 5.25 & 3.52 & 2.09 & \\
\hline 06226-0905 & 50707.0 & 5.15 & 3.44 & 2.07 & 0.51 \\
\hline 06226-0905 & 50908.0 & 4.44 & 2.84 & 1.58 & 0.09 \\
\hline 06226-0905 & 51145.1 & 5.76 & 4.00 & 2.51 & \\
\hline $06268+0849$ & 50407.0 & 8.41 & 6.30 & 4.49 & 1.94 \\
\hline $06268+0849$ & 50690.0 & 8.85 & 6.67 & 4.74 & \\
\hline $06268+0849$ & 50709.0 & 8.78 & 6.62 & 4.72 & 2.12 \\
\hline $06268+0849$ & 50811.0 & 8.70 & 6.90 & 4.80 & \\
\hline $06268+0849$ & 50877.0 & 8.03 & 5.94 & 4.17 & 1.53 \\
\hline $06268+0849$ & 51145.1 & 9.54 & 7.34 & 5.26 & \\
\hline $06487+0551$ & 50408.0 & 8.37 & 6.19 & 4.30 & 1.87 \\
\hline $06487+0551$ & 50690.0 & 9.30 & 6.88 & 4.74 & \\
\hline $06487+0551$ & 50709.0 & 9.42 & 7.04 & 5.03 & 2.46 \\
\hline $06487+0551$ & 50809.0 & 9.51 & 7.03 & 4.99 & \\
\hline $06487+0551$ & 51145.1 & 8.26 & 6.08 & 4.21 & \\
\hline $06487+0551$ & 51292.8 & 9.20 & 6.81 & 5.02 & \\
\hline $06531-0216$ & 50407.0 & 5.99 & 4.29 & 2.90 & 1.11 \\
\hline 06531-0216 & 50497.0 & 6.55 & 4.83 & 3.31 & \\
\hline $06531-0216$ & 50707.0 & 6.90 & 5.06 & 3.52 & \\
\hline 06531-0216 & 50811.0 & 7.43 & 5.75 & 4.32 & \\
\hline 06531-0216 & 51145.1 & 7.57 & 5.70 & 3.99 & \\
\hline 06531-0216 & 51292.9 & 7.13 & 5.11 & 3.67 & \\
\hline $17581-1744$ & 50268.0 & 7.12 & 5.15 & 3.42 & 1.28 \\
\hline $17581-1744$ & 50303.0 & 6.98 & 4.93 & 3.32 & \\
\hline $17581-1744$ & 50642.0 & 8.61 & 6.47 & 4.61 & 2.06 \\
\hline 17581-1744 & 50690.0 & 8.31 & 6.13 & 4.32 & \\
\hline 17581-1744 & 50692.0 & 8.33 & 6.13 & 4.32 & \\
\hline $17581-1744$ & 50708.0 & 8.37 & 6.16 & 4.36 & 2.08 \\
\hline $17581-1744$ & 50908.0 & 7.42 & 5.30 & 3.58 & 1.31 \\
\hline 17581-1744 & 50947.0 & 7.53 & 5.35 & 3.63 & 1.36 \\
\hline $17581-1744$ & 51292.2 & 8.80 & 6.31 & 4.37 & \\
\hline $17581-1744$ & 51393.0 & 7.45 & 5.24 & 3.53 & \\
\hline 18040-0941 & 50268.0 & 6.61 & 3.38 & 1.75 & 0.30 \\
\hline 18040-0941 & 50303.0 & 5.61 & 3.55 & 1.91 & \\
\hline 18040-0941 & 50643.0 & 6.90 & 4.71 & 2.92 & 0.52 \\
\hline $18040-0941$ & 50691.0 & 6.64 & 4.39 & 2.59 & \\
\hline $18040-0941$ & 50709.0 & 6.52 & 4.30 & 2.56 & 0.26 \\
\hline 18040-0941 & 50985.0 & 6.19 & 3.91 & 2.18 & -0.06 \\
\hline $18040-0941$ & 51293.1 & 6.97 & 4.60 & 2.65 & \\
\hline 18040-0941 & 51393.9 & 5.98 & 3.72 & 1.95 & \\
\hline $18239-0655$ & 50268.0 & 11.16 & & & 1.92 \\
\hline $18239-0655$ & 50303.0 & & 8.31 & 5.65 & \\
\hline $18239-0655$ & 50303.0 & & 8.22 & 5.55 & \\
\hline $18239-0655$ & 50643.0 & 9.40 & 6.80 & 4.30 & 1.00 \\
\hline $18239-0655$ & 50690.0 & 9.44 & 6.72 & 4.18 & \\
\hline
\end{tabular}


F. Kerschbaum et al.: Near-infrared variability of a sample of galactic carbon Miras, Online Material p 5

Table A.2. continued.

\begin{tabular}{|c|c|c|c|c|c|}
\hline$\overline{\text { IRAS }}$ & MJD & $J$ & $\bar{H}$ & $\bar{K}$ & $\overline{L^{\prime}}$ \\
\hline $18239-0655$ & 50692.0 & 9.38 & 6.71 & 4.18 & \\
\hline $18239-0655$ & 50947.0 & 10.58 & 7.96 & 5.34 & 1.73 \\
\hline $18239-0655$ & 51293.1 & 9.27 & 6.45 & 3.91 & \\
\hline $18239-0655$ & 51394.0 & 10.11 & 7.26 & 4.62 & \\
\hline $18239-0655$ & 51394.9 & 10.19 & 7.26 & 4.63 & \\
\hline $18240+2326$ & 50265.0 & 11.13 & 8.34 & 5.17 & 0.73 \\
\hline $18240+2326$ & 50303.0 & & 8.10 & 4.96 & \\
\hline $18240+2326$ & 50643.0 & 13.00 & 10.22 & 6.88 & 2.04 \\
\hline $18240+2326$ & 50692.0 & 11.80 & 10.19 & 6.83 & \\
\hline $18240+2326$ & 50709.0 & 12.90 & 9.95 & 6.70 & 1.98 \\
\hline $18240+2326$ & 50947.0 & 11.14 & 8.38 & 5.23 & 0.74 \\
\hline $18240+2326$ & 51086.9 & 11.60 & 8.93 & 5.65 & 1.12 \\
\hline $18240+2326$ & 51292.1 & 12.10 & 10.50 & 7.04 & \\
\hline $18244-0815$ & 50268.0 & 10.32 & & & 2.39 \\
\hline $18244-0815$ & 50303.0 & & 7.42 & 5.12 & \\
\hline $18244-0815$ & 50643.0 & & 9.61 & 6.44 & 3.13 \\
\hline $18244-0815$ & 50691.0 & & 8.96 & 6.35 & \\
\hline $18244-0815$ & 50709.0 & 10.55 & 8.93 & 6.36 & 3.20 \\
\hline $18244-0815$ & 50908.0 & 11.00 & 7.83 & 5.30 & 2.37 \\
\hline $18244-0815$ & 50947.0 & 10.79 & 7.94 & 5.47 & 2.45 \\
\hline $18244-0815$ & 51292.2 & 12.50 & 9.40 & 6.39 & \\
\hline $18248-0839$ & 50268.0 & 12.60 & & & 2.50 \\
\hline $18248-0839$ & 50303.0 & & & 6.95 & \\
\hline $18248-0839$ & 50303.0 & & & 6.95 & \\
\hline $18248-0839$ & 50643.0 & 11.70 & 8.60 & 5.60 & 1.60 \\
\hline $18248-0839$ & 50690.0 & & 8.45 & 5.37 & \\
\hline $18248-0839$ & 50692.0 & 12.02 & 8.43 & 5.37 & \\
\hline $18248-0839$ & 50709.0 & 11.95 & 8.40 & 5.34 & 1.44 \\
\hline $18248-0839$ & 50947.0 & 13.20 & 10.13 & 6.91 & 2.54 \\
\hline $18248-0839$ & 51293.1 & 11.60 & 8.75 & 5.64 & \\
\hline $18269-1257$ & 50303.0 & & & 7.95 & \\
\hline $18269-1257$ & 50644.0 & & 9.42 & 5.73 & 1.94 \\
\hline 18269-1257 & 50692.0 & 15.40 & 8.99 & 5.81 & \\
\hline 18269-1257 & 50947.0 & 12.40 & 10.30 & 9.28 & \\
\hline $18269-1257$ & 51293.2 & & 9.64 & 6.24 & \\
\hline 18269-1257 & 51394.9 & & 9.52 & 5.80 & \\
\hline $18320-0352$ & 50303.0 & & & 7.54 & \\
\hline $18320-0352$ & 50644.0 & & 12.00 & 9.10 & 4.70 \\
\hline $18320-0352$ & 50691.0 & & & 8.98 & \\
\hline $18320-0352$ & 50985.0 & 13.80 & 10.71 & 7.56 & 3.18 \\
\hline 18320-0352 & 51086.8 & 13.20 & 11.06 & 7.96 & 3.95 \\
\hline $18320-0352$ & 51294.1 & 12.00 & 11.30 & 8.75 & \\
\hline $18367-0452$ & 50303.0 & & & 8.82 & \\
\hline $18367-0452$ & 50644.0 & 12.40 & 10.23 & 7.34 & 2.77 \\
\hline $18367-0452$ & 50690.0 & & 10.49 & 7.59 & \\
\hline $18367-0452$ & 50692.0 & 12.45 & 10.47 & 7.59 & \\
\hline $18367-0452$ & 50948.0 & 11.69 & 10.67 & 8.83 & 4.25 \\
\hline $18367-0452$ & 50985.0 & 12.50 & 10.94 & 8.77 & 4.05 \\
\hline $18367-0452$ & 51086.8 & 12.10 & 10.65 & 7.72 & 4.00 \\
\hline $18367-0452$ & 51294.1 & 11.60 & 9.88 & 7.30 & \\
\hline $18397+1738$ & 50265.0 & 4.72 & 2.83 & 1.17 & -1.23 \\
\hline $18397+1738$ & 50303.0 & 4.60 & 2.78 & 1.15 & \\
\hline $18397+1738$ & 50303.0 & 4.52 & 2.70 & 1.05 & \\
\hline $18397+1738$ & 50643.0 & 5.62 & 3.80 & 2.08 & -0.49 \\
\hline $18397+1738$ & 50692.0 & 5.05 & 3.18 & 1.44 & \\
\hline $18397+1738$ & 50708.0 & 4.93 & 3.06 & 1.34 & -1.10 \\
\hline $18397+1738$ & 50908.0 & 5.85 & 3.79 & 1.93 & -0.67 \\
\hline $18397+1738$ & 50947.0 & 6.18 & 4.12 & 2.23 & -0.43 \\
\hline $18397+1738$ & 50986.0 & 6.48 & 4.40 & 2.53 & -0.21 \\
\hline $18397+1738$ & 51086.9 & 6.42 & 4.29 & 2.31 & -0.27 \\
\hline
\end{tabular}


F. Kerschbaum et al.: Near-infrared variability of a sample of galactic carbon Miras, Online Material p 6

Table A.2. continued.

\begin{tabular}{|c|c|c|c|c|c|}
\hline$\overline{\text { IRAS }}$ & MJD & $\bar{J}$ & $\bar{H}$ & $\bar{K}$ & $\overline{L^{\prime}}$ \\
\hline $18397+1738$ & 51292.1 & 5.01 & 2.95 & 1.19 & \\
\hline $18398-0220$ & 50303.0 & 4.98 & 2.94 & 1.31 & \\
\hline $18398-0220$ & 50303.0 & 4.98 & 3.00 & 1.41 & \\
\hline $18398-0220$ & 50643.0 & 5.79 & 3.76 & 2.08 & -0.12 \\
\hline $18398-0220$ & 50691.0 & 5.53 & 3.46 & 1.83 & \\
\hline $18398-0220$ & 50947.0 & 4.81 & 2.83 & 1.25 & -0.97 \\
\hline $18398-0220$ & 51085.8 & 5.88 & 3.66 & 1.83 & \\
\hline $18398-0220$ & 51294.2 & 6.26 & 4.05 & 2.21 & \\
\hline $18424+0346$ & 50303.0 & 8.36 & 5.84 & 3.82 & \\
\hline $18424+0346$ & 50303.0 & 8.38 & 5.72 & 3.73 & \\
\hline $18424+0346$ & 50643.0 & 9.16 & 6.70 & 4.58 & 1.78 \\
\hline $18424+0346$ & 50690.0 & 8.62 & 6.13 & 4.03 & \\
\hline $18424+0346$ & 50692.0 & 8.53 & 6.08 & 4.02 & \\
\hline $18424+0346$ & 50975.0 & 10.27 & 7.92 & 5.77 & \\
\hline $18424+0346$ & 51086.9 & 9.76 & 7.35 & 5.23 & 2.73 \\
\hline $18424+0346$ & 51292.2 & 9.09 & 6.62 & 4.85 & \\
\hline $18475+0926$ & 50265.0 & 11.29 & 8.80 & 5.89 & 1.73 \\
\hline $18475+0926$ & 50303.0 & & 8.90 & 6.02 & \\
\hline $18475+0926$ & 50303.0 & & 8.82 & 5.74 & \\
\hline $18475+0926$ & 50643.0 & 11.80 & 9.79 & 7.22 & 2.78 \\
\hline $18475+0926$ & 50692.0 & 12.10 & 9.80 & 7.16 & \\
\hline $18475+0926$ & 50948.0 & 11.08 & 8.29 & 5.59 & 1.48 \\
\hline $18475+0926$ & 50975.0 & 10.80 & 8.35 & 5.61 & \\
\hline $18475+0926$ & 50985.0 & 11.10 & 8.32 & 5.58 & 1.53 \\
\hline $18475+0926$ & 51085.9 & 11.39 & 8.78 & 6.05 & \\
\hline $18475+0926$ & 51294.1 & 12.10 & 10.10 & 7.23 & \\
\hline $19029+0808$ & 50265.0 & 11.38 & 8.46 & 5.44 & 1.53 \\
\hline $19029+0808$ & 50303.0 & & 8.72 & 5.54 & \\
\hline $19029+0808$ & 50643.0 & 12.00 & 9.81 & 7.00 & 2.45 \\
\hline $19029+0808$ & 50691.0 & & 9.41 & 6.23 & \\
\hline $19029+0808$ & 50948.0 & 10.94 & 8.03 & 5.05 & 1.16 \\
\hline $19029+0808$ & 50975.0 & & 8.52 & 5.22 & \\
\hline $19029+0808$ & 50985.0 & 11.70 & 8.40 & 5.26 & 1.35 \\
\hline $19029+0808$ & 51085.9 & 12.02 & 9.20 & 5.99 & \\
\hline $19029+0808$ & 51294.2 & 10.06 & 8.89 & 7.18 & \\
\hline $19029+0808$ & 51393.0 & 12.00 & 9.89 & 6.81 & \\
\hline $19029+2017$ & 50643.0 & 7.54 & 5.41 & 3.64 & 2.45 \\
\hline $19029+2017$ & 50690.0 & 7.21 & 5.06 & 3.33 & \\
\hline $19029+2017$ & 50692.0 & 7.15 & 5.03 & 3.30 & \\
\hline $19029+2017$ & 50908.0 & 8.02 & 5.77 & 3.90 & 1.45 \\
\hline $19029+2017$ & 50985.0 & 8.59 & 6.28 & 4.35 & 1.95 \\
\hline $19029+2017$ & 51294.1 & 7.15 & 5.04 & 3.30 & \\
\hline $19068+0544$ & 50303.0 & 7.67 & 5.38 & 3.62 & \\
\hline $19068+0544$ & 50303.0 & 7.80 & 5.54 & 3.80 & \\
\hline $19068+0544$ & 50692.0 & 8.02 & 5.74 & 4.04 & \\
\hline $19068+0544$ & 50948.0 & 7.19 & 4.92 & 3.46 & 1.54 \\
\hline $19068+0544$ & 50985.0 & 7.59 & 5.30 & 3.68 & 1.76 \\
\hline $19068+0544$ & 51085.9 & 8.67 & 6.24 & 4.39 & \\
\hline $19068+0544$ & 51294.2 & 8.03 & 5.87 & 4.34 & \\
\hline $19068+0544$ & 51395.0 & 6.76 & 4.63 & 3.20 & \\
\hline $19175-0807$ & 50265.0 & 7.96 & 5.39 & 3.14 & 0.17 \\
\hline $19175-0807$ & 50303.0 & 7.21 & 4.72 & 2.97 & \\
\hline $19175-0807$ & 50643.0 & 7.91 & 5.28 & 3.05 & 0.13 \\
\hline 19175-0807 & 50691.0 & 8.30 & 5.62 & 3.34 & \\
\hline $19175-0807$ & 50707.0 & 8.27 & 5.61 & 3.36 & 0.51 \\
\hline $19175-0807$ & 50948.0 & 7.20 & 4.72 & 2.67 & -0.08 \\
\hline $19175-0807$ & 50975.0 & 6.80 & 4.33 & 2.29 & \\
\hline $19175-0807$ & 50985.0 & 6.56 & 4.05 & 2.06 & -0.50 \\
\hline $19175-0807$ & 51085.8 & 5.86 & 3.52 & 1.64 & \\
\hline $19175-0807$ & 51294.2 & 6.72 & 4.45 & 2.73 & \\
\hline
\end{tabular}


F. Kerschbaum et al.: Near-infrared variability of a sample of galactic carbon Miras, Online Material $p 7$

Table A.2. continued.

\begin{tabular}{|c|c|c|c|c|c|}
\hline IRAS & MJD & $J$ & $\bar{H}$ & $\bar{K}$ & $L^{\prime}$ \\
\hline $19455+0920$ & 50268.0 & 12.64 & & & 3.74 \\
\hline $19455+0920$ & 50303.0 & & & 7.49 & \\
\hline $19455+0920$ & 50642.0 & 11.97 & 9.75 & 6.70 & 3.03 \\
\hline $19455+0920$ & 50692.0 & 12.47 & 9.68 & 7.07 & \\
\hline $19455+0920$ & 51085.9 & 11.10 & 8.35 & 5.81 & \\
\hline $19455+0920$ & 51146.8 & 11.80 & 8.72 & 6.11 & \\
\hline $19455+0920$ & 51293.2 & 12.20 & 10.15 & 7.67 & \\
\hline $19455+0920$ & 51394.0 & 12.60 & 9.95 & 7.30 & \\
\hline $19548+3035$ & 50268.0 & 10.28 & & & 4.96 \\
\hline $19548+3035$ & 50303.0 & & 8.90 & 8.44 & \\
\hline $19548+3035$ & 50303.0 & & 8.88 & 8.38 & \\
\hline $19548+3035$ & 50644.0 & 10.16 & 8.95 & 8.49 & 6.60 \\
\hline $19548+3035$ & 50691.0 & 10.13 & 8.92 & 8.53 & \\
\hline $19548+3035$ & 51393.0 & 10.25 & 9.03 & 8.44 & \\
\hline $20072+3116$ & 49967.0 & 7.22 & 4.92 & 3.19 & 0.86 \\
\hline $20072+3116$ & 50690.0 & 6.81 & 4.61 & 2.82 & \\
\hline $20072+3116$ & 50692.0 & 6.78 & 4.61 & 2.83 & \\
\hline $20072+3116$ & 50709.0 & 6.76 & 4.56 & 2.78 & 0.31 \\
\hline $20072+3116$ & 50908.0 & 6.98 & 4.74 & 2.93 & 0.37 \\
\hline $20072+3116$ & 50985.0 & 7.57 & 5.28 & 3.41 & 0.85 \\
\hline $20072+3116$ & 51145.8 & 8.70 & 6.33 & 4.35 & \\
\hline $20072+3116$ & 51293.2 & 8.24 & 6.10 & 3.87 & \\
\hline $20072+3116$ & 51394.1 & 7.07 & 4.85 & 3.00 & \\
\hline $20082+3228$ & 50303.0 & & 7.51 & 5.19 & \\
\hline $20082+3228$ & 50644.0 & 11.80 & 9.15 & 6.58 & 3.11 \\
\hline $20082+3228$ & 50692.0 & 12.10 & 9.24 & 6.61 & \\
\hline $20082+3228$ & 50985.0 & 11.27 & 8.12 & 5.62 & 2.45 \\
\hline $20082+3228$ & 51146.8 & 12.10 & 9.54 & 6.91 & \\
\hline $20082+3228$ & 51395.0 & 11.78 & 8.80 & 6.20 & \\
\hline $20171+3519$ & 50303.0 & & & 8.76 & \\
\hline $20171+3519$ & 50643.0 & & 10.20 & 7.00 & 3.00 \\
\hline $20171+3519$ & 50985.0 & 12.90 & 13.00 & 9.00 & 4.40 \\
\hline $20171+3519$ & 51145.9 & 10.50 & 9.04 & 8.47 & \\
\hline $20171+3519$ & 51293.2 & 13.20 & 11.80 & 8.09 & \\
\hline $20435+3825$ & 50265.0 & 11.21 & 8.28 & 5.88 & 1.96 \\
\hline $20435+3825$ & 50303.0 & & 8.39 & 5.67 & \\
\hline $20435+3825$ & 50642.0 & 12.03 & 9.00 & 6.25 & 2.64 \\
\hline $20435+3825$ & 50692.0 & 11.36 & 8.45 & 5.78 & \\
\hline $20435+3825$ & 50707.0 & 12.01 & 8.91 & 6.24 & 2.73 \\
\hline $20435+3825$ & 50986.0 & 11.90 & 8.91 & 6.11 & 2.48 \\
\hline $20435+3825$ & 51145.9 & 11.70 & 8.90 & 6.30 & \\
\hline $20435+3825$ & 51293.3 & 11.70 & 8.09 & 5.55 & \\
\hline $20435+3825$ & 51395.1 & 10.25 & 7.48 & 5.07 & \\
\hline $20532+5554$ & 50303.0 & & 8.55 & 5.87 & \\
\hline $20532+5554$ & 50303.0 & & 8.63 & 5.93 & \\
\hline $20532+5554$ & 50303.0 & & 8.76 & 6.12 & \\
\hline $20532+5554$ & 50642.0 & 11.82 & 9.20 & 6.37 & 2.60 \\
\hline $20532+5554$ & 50691.0 & & 8.63 & 5.93 & \\
\hline $20532+5554$ & 50809.0 & 11.20 & 8.19 & 5.35 & \\
\hline $20532+5554$ & 50986.0 & 12.20 & 9.21 & 6.34 & 2.57 \\
\hline $20532+5554$ & 51146.9 & 13.00 & 9.96 & 7.17 & \\
\hline $20532+5554$ & 51294.2 & 12.00 & 9.10 & 6.56 & \\
\hline $20570+2714$ & 49968.0 & 9.68 & 7.01 & 4.56 & 0.98 \\
\hline $20570+2714$ & 50266.0 & 9.92 & & & 0.92 \\
\hline $20570+2714$ & 50303.0 & 9.07 & 6.38 & 3.90 & \\
\hline $20570+2714$ & 50642.0 & 9.96 & 7.17 & 4.55 & 0.99 \\
\hline $20570+2714$ & 50692.0 & 10.30 & 7.70 & 5.01 & \\
\hline $20570+2714$ & 50709.0 & 10.51 & 7.83 & 5.14 & 1.35 \\
\hline $20570+2714$ & 50986.0 & 9.15 & 6.38 & 3.61 & 0.21 \\
\hline
\end{tabular}


F. Kerschbaum et al.: Near-infrared variability of a sample of galactic carbon Miras, Online Material $p 8$

Table A.2. continued.

\begin{tabular}{|c|c|c|c|c|c|}
\hline$\overline{\text { IRAS }}$ & MJD & $\bar{J}$ & $\bar{H}$ & $\bar{K}$ & $\overline{L^{\prime}}$ \\
\hline $20570+2714$ & 51146.9 & 8.52 & 5.83 & 3.32 & \\
\hline $20570+2714$ & 51394.1 & 10.40 & 7.99 & 5.28 & \\
\hline $21003+4801$ & 50303.0 & & 8.59 & 6.11 & \\
\hline $21003+4801$ & 50643.0 & 9.86 & 7.14 & 4.67 & 1.46 \\
\hline $21003+4801$ & 50644.0 & 9.93 & 7.19 & 4.72 & 1.42 \\
\hline $21003+4801$ & 50692.0 & 10.10 & 7.36 & 4.90 & \\
\hline $21003+4801$ & 50708.0 & 10.22 & 7.48 & 5.01 & 1.75 \\
\hline $21003+4801$ & 50986.0 & 10.61 & 8.21 & 5.64 & 2.31 \\
\hline $21003+4801$ & 51145.9 & 11.00 & 8.20 & 5.69 & \\
\hline $21027+5309$ & 49972.0 & 8.96 & 6.54 & 4.48 & 1.81 \\
\hline $21027+5309$ & 50268.0 & 10.20 & & & 2.42 \\
\hline $21027+5309$ & 50303.0 & & 8.00 & 5.76 & \\
\hline $21027+5309$ & 50408.0 & 9.74 & 7.35 & 5.24 & \\
\hline $21027+5309$ & 50643.0 & 9.02 & 6.64 & 4.47 & 1.67 \\
\hline $21027+5309$ & 50691.0 & 9.33 & 6.93 & 4.82 & \\
\hline $21027+5309$ & 50707.0 & 9.56 & 7.12 & 4.95 & 2.07 \\
\hline $21027+5309$ & 50986.0 & 10.50 & 8.08 & 5.67 & 2.58 \\
\hline $21027+5309$ & 51146.9 & 9.22 & 6.98 & 4.82 & \\
\hline $21147+5110$ & 50268.0 & 11.93 & & & 4.13 \\
\hline $21147+5110$ & 50303.0 & & & 9.25 & \\
\hline $21147+5110$ & 50408.0 & & 11.23 & 9.42 & \\
\hline $21147+5110$ & 50643.0 & 13.70 & 11.29 & 7.91 & 3.31 \\
\hline $21147+5110$ & 50690.0 & & 10.65 & 7.57 & \\
\hline $21147+5110$ & 50691.0 & 13.04 & 10.74 & 7.54 & \\
\hline $21147+5110$ & 50708.0 & 12.80 & 10.70 & 7.49 & 3.13 \\
\hline $21147+5110$ & 50986.0 & 12.70 & 11.49 & 8.64 & 3.99 \\
\hline $21147+5110$ & 51147.0 & 12.50 & 12.36 & 9.26 & \\
\hline $21223+5114$ & 50303.0 & & & 8.13 & \\
\hline $21223+5114$ & 50644.0 & 12.70 & 9.74 & 6.79 & 2.93 \\
\hline $21223+5114$ & 50692.0 & 13.30 & 9.92 & 6.96 & \\
\hline $21223+5114$ & 50986.0 & & 10.55 & 7.40 & 3.78 \\
\hline $21223+5114$ & 51146.9 & 11.90 & 9.10 & 6.34 & \\
\hline $21223+5114$ & 51394.1 & 14.00 & 9.68 & 6.98 & \\
\hline $21373+4540$ & 50303.0 & & 9.53 & 7.02 & \\
\hline $21373+4540$ & 50643.0 & 10.67 & 7.88 & 5.46 & 2.13 \\
\hline $21373+4540$ & 50691.0 & & 8.16 & 5.78 & \\
\hline $21373+4540$ & 50707.0 & 11.14 & 8.21 & 5.82 & 2.43 \\
\hline $21373+4540$ & 50986.0 & & 9.62 & 6.87 & 3.29 \\
\hline $21373+4540$ & 51086.0 & 11.30 & 8.93 & 6.09 & \\
\hline $21373+4540$ & 51146.0 & 10.40 & 7.83 & 5.63 & \\
\hline $21373+4540$ & 51394.2 & 11.40 & 8.53 & 6.22 & \\
\hline $21449+4950$ & 50303.0 & & 9.03 & 6.62 & \\
\hline $21449+4950$ & 50643.0 & 11.30 & 8.45 & 6.06 & 2.97 \\
\hline $21449+4950$ & 50690.0 & & 8.20 & 5.90 & \\
\hline $21449+4950$ & 50692.0 & 10.97 & 8.17 & 5.87 & \\
\hline $21449+4950$ & 50809.0 & 10.83 & 8.08 & 5.59 & \\
\hline $21449+4950$ & 50986.0 & 11.85 & 9.21 & 6.51 & 3.38 \\
\hline $21449+4950$ & 51147.0 & 11.80 & 9.06 & 6.63 & \\
\hline $21449+4950$ & 51395.1 & 10.53 & 7.73 & 5.47 & \\
\hline $22241+6005$ & 50265.0 & 10.27 & 7.24 & 4.63 & 1.15 \\
\hline $22241+6005$ & 50303.0 & & 7.68 & 4.99 & \\
\hline $22241+6005$ & 50644.0 & 9.14 & 6.47 & 4.03 & 0.87 \\
\hline $22241+6005$ & 50690.0 & 8.82 & 6.14 & 3.77 & \\
\hline $22241+6005$ & 50692.0 & 8.81 & 6.15 & 3.76 & \\
\hline $22241+6005$ & 50809.0 & 9.03 & 6.31 & 3.79 & \\
\hline $22241+6005$ & 50811.0 & 8.84 & 6.54 & 3.80 & \\
\hline $22241+6005$ & 50986.0 & & & & 1.62 \\
\hline $22241+6005$ & 51087.1 & 10.41 & 7.54 & 5.17 & 2.14 \\
\hline $22241+6005$ & 51146.0 & 9.61 & 6.89 & 4.55 & \\
\hline
\end{tabular}


F. Kerschbaum et al.: Near-infrared variability of a sample of galactic carbon Miras, Online Material p 9

Table A.2. continued.

\begin{tabular}{ccrccc}
\hline \hline IRAS & \multicolumn{1}{c}{ MJD } & \multicolumn{1}{c}{$J$} & \multicolumn{1}{c}{$H$} & \multicolumn{1}{c}{$K$} & \multicolumn{1}{c}{$L^{\prime}$} \\
\hline $23174+5941$ & 50265.0 & 9.25 & 6.92 & 4.99 & 2.45 \\
$23174+5941$ & 50303.0 & 9.13 & 6.87 & 4.95 & \\
$23174+5941$ & 50407.0 & 9.01 & 6.74 & 4.84 & 2.42 \\
$23174+5941$ & 50644.0 & 10.25 & 7.91 & 5.87 & 3.35 \\
$23174+5941$ & 50691.0 & 10.20 & 7.84 & 5.78 & \\
$23174+5941$ & 50707.0 & 10.10 & 7.77 & 5.74 & 3.27 \\
$23174+5941$ & 50809.0 & 9.01 & 6.69 & 4.67 & \\
$23174+5941$ & 51147.0 & 9.72 & 7.52 & 5.63 & \\
$23174+5941$ & 51395.1 & 8.15 & 6.09 & 4.44 & \\
& & & & & \\
$23279+5336$ & 50268.0 & 10.40 & & & 2.81 \\
$23279+5336$ & 50303.0 & & 8.20 & 6.02 & \\
$23279+5336$ & 50303.0 & & 8.44 & 6.09 & \\
$23279+5336$ & 50407.0 & 10.46 & 8.03 & 5.82 & 2.93 \\
$23279+5336$ & 50644.0 & 9.12 & 6.71 & 4.64 & 3.35 \\
$23279+5336$ & 50690.0 & 9.57 & & 4.98 & \\
$23279+5336$ & 50692.0 & 9.52 & & 5.00 & \\
$23279+5336$ & 50707.0 & 9.72 & 7.27 & 5.14 & 2.39 \\
$23279+5336$ & 51085.1 & 9.09 & 7.03 & 5.16 & 1.89 \\
\hline
\end{tabular}

